\title{
Evaluation of the yield and nitrogen use efficiency of the dominant maize hybrids grown in North and Northeast China
}

\author{
CHEN FanJun $^{1}$, FANG ZenGuo ${ }^{2}$, GAO Qiang $^{3}$, YE YouLiang ${ }^{4}$, JIA LiangLiang $^{5}$, \\ YUAN LiXing $^{1}$, MI GuoHua $^{1 *}$ \& ZHANG FuSuo ${ }^{1}$ \\ ${ }^{1}$ Key Laboratory of Plant-Soil Interaction, Ministry of Education; College of Resources and Environmental Science, \\ China Agricultural University, Beijing 100193, China; \\ ${ }^{2}$ College of Resources and Environmental Science, Qingdao Agricultural University, Qingdao 266109, China; \\ ${ }^{3}$ College of Resources and Environmental Science, Jilin Agricultural University, Changchun 130118, China; \\ ${ }^{4}$ College of Resources and Environmental Science, Henan Agricultural University, Zhengzhou 450002, China; \\ ${ }^{5}$ Institute of Agricultural Resources and Environment, Hebei Academy of Agriculture and Forestry, Shijiazhuang 050051, China
}

Received July 12, 2012; accepted January 10, 2013; published online March 15, 2013

\begin{abstract}
Breeding high-yielding and nutrient-efficient cultivars is one strategy to simultaneously resolve the problems of food security, resource shortage, and environmental pollution. However, the potential increased yield and reduction in fertilizer input achievable by using high-yielding and nutrient-efficient cultivars is unclear. In the present study, we evaluated the yield and nitrogen use efficiency (NUE) of 40 commercial maize hybrids at five locations in North and Northeast China in 2008 and 2009. The effect of interaction between genotype and nitrogen $(\mathrm{N})$ input on maize yield was significant when the yield reduction under low-N treatment was $25 \%-60 \%$. Based on the average yields achieved with high or low $\mathrm{N}$ application, the tested cultivars were classified into four types based on their NUE: efficient-efficient (EE) were efficient under both low and high $\mathrm{N}$ inputs, high-N efficient (HNE) under only high $\mathrm{N}$ input, low-N efficient (LNE) under only low $\mathrm{N}$ input, and nonefficient-nonefficient under neither low nor high $\mathrm{N}$ inputs. Under high $\mathrm{N}$ application, EE and HNE cultivars could potentially increase maize yield by $8 \%-10 \%$ and reduce $\mathrm{N}$ input by $16 \%-21 \%$. Under low N application, LNE cultivars could potentially increase maize yield by $12 \%$. We concluded that breeding for N-efficient cultivars is a feasible strategy to increase maize yield and/or reduce $\mathrm{N}$ input.
\end{abstract}

maize, genotypexnitrogen interaction, low nitrogen stress, nitrogen use efficiency, yield

Citation: Chen F J, Fang Z G, Gao Q, et al. Evaluation of the yield and nitrogen use efficiency of the dominant maize hybrids grown in North and Northeast China. Sci China Life Sci, 2013, 56: 552-560, doi: 10.1007/s11427-013-4462-8

Food security is among the most important concerns in China. In 2011, the Food Security Risk Index of China was predicted as 'medium risk' by the FAO and Maplecroft in the United Kingdom [1]. To meet the food requirements of China's increasing population, estimates indicate that the crop yield per unit area should be increased to as high as $5250 \mathrm{~kg} \mathrm{hm}^{-2}$ by 2020 [2]. However, fertilizer application is increasing rapidly in China and has reached a total of 50

*Corresponding author (email: miguohua@ cau.edu.cn) million tons per annum [3]. Overuse of fertilizer not only reduces fertilizer efficiency, but also increases soil nutrient loss and results in environmental pollution. Methods to reduce fertilizer input while maintaining or even increasing crop yield are a major goal of crop research [4,5].

Among crops in China, maize has the highest total yield and growing area. The partial fertilizer productivity (PFP) of nitrogen ( $\mathrm{N}$ ) in China is only $21-38 \mathrm{~kg} \mathrm{~kg}^{-1}$ for maize [6-11], whereas the average PFP worldwide is as high as 57 $\mathrm{kg} \mathrm{kg}^{-1}$ [11]. Therefore, there is substantial potential to in- 
crease nitrogen use efficiency (NUE) in maize production in China. Breeding for $\mathrm{N}$-efficient cultivars may contribute to higher NUE in addition to higher yield. Variation in NUE among maize genotypes is well documented [6,12-14], providing opportunities for genetic improvement of this trait. Breeding for low-N-tolerant maize has long been a target of International Maize and Wheat Improvement Center (CIMMYT) [15]. Some major maize-breeding companies, such as Pioneer, also rate NUE among the highest priorities of their breeding programs [16]. As one example of success, $\mathrm{N}$-efficient maize cultivars bred in a low-N environment showed increased yield of $10.5 \%$ under high-N conditions and $14 \%$ under low-N conditions. Therefore, increasing maize NUE under low-N supply while maintaining the yield potential under high-N conditions is feasible [17].

The targets for NUE improvement are to (i) increase yield potential without additional $\mathrm{N}$ input, (ii) reduce $\mathrm{N}$ input without affecting yield significantly, or (iii) increase low-N tolerance with very low $\mathrm{N}$ input [18]. In Africa, where the population is less dense than in China and where $\mathrm{N}$ fertilizer supply is limited, low-N-tolerant cultivars are highly desirable [19]. In China, where the population is large and where food shortages and environmental pollution are urgent problems, cultivars must be bred with higher yields and low N-input requirements. Liu et al. [20] classified cultivars into four different NUE classes based on the conditions under which they are efficient: efficient-efficient (EE) cultivars are efficient under both low and high nitrogen inputs; high-nitrogen efficient (HNE) cultivars under only high nitrogen input; low-nitrogen efficient (LNE) cultivars under only low nitrogen input; and nonefficientnonefficient (NN) cultivars under neither. The extent of variation in NUE among the dominant Chinese hybrids in cultivation and the potential to increase yield and reduce $\mathrm{N}$ fertilizer input through high-yielding, N-efficient cultivars is unclear. In the present study, we evaluated the yield and NUE of 15 commercial maize hybrids at five locations in North and Northeast China. The results are important for maize breeders to set targets for increasing NUE.

\section{Materials and methods}

\subsection{Experimental locations}

Ten to 15 maize hybrids comprising the dominant cultivars grown in North and/or Northeast China were grown in each of the eight environments at five locations in China in 2008 and 2009: Changping, Beijing; Changchun, Jilin Province; Qingdao, Shangdong Province; Xuchang, Henan Province; and Hengshui, Hebei Province (Tables 1 and 2). The growth period was from early May to the end of September in Beijing and Changchun, and from mid-June to mid-October in Qingdao, Xuchang, and Hengshui. The soil physicochemical characteristics at the onset of the experiment are shown in Table 2. The experimental field at Changping has been part of a long-term $\mathrm{N}$ fertilizer experiment since 1984 [21].

\subsection{Experimental design}

The experimental design was a split-plot with three replicates, with $\mathrm{N}$ fertilizer treatments in the main plots and the cultivars in the subplots. The plots were $12-20 \mathrm{~m}^{2}$ in area. The rows were $6 \mathrm{~m}$ long and spaced 50-60 cm apart. Seeds were hand-sown at a density of 60000 seeds $\mathrm{hm}^{-2}$. The distance between plants within a row was $0.28-0.33 \mathrm{~m}$. For the high $\mathrm{N}(\mathrm{HN})$, medium $\mathrm{N}(\mathrm{MN})$, and low $\mathrm{N}(\mathrm{LN})$ treatments, 240,120 , and $0 \mathrm{~kg} \mathrm{~N} \mathrm{hm}^{-2}$ (as urea), respectively, were applied, half at sowing and half at the V12 stage. Potassium (as $\mathrm{K}_{2} \mathrm{SO}_{4}$ ) and phosphorus (as superphosphate) were applied before sowing at $30-45 \mathrm{~kg} \mathrm{~K}_{2} \mathrm{O} \mathrm{hm}{ }^{-2}$ and $60-90 \mathrm{~kg}$

Table 1 Maize cultivars tested at each location in China

\begin{tabular}{|c|c|c|c|c|}
\hline \multicolumn{5}{|c|}{ Location } \\
\hline Xuchang, Henan & Hengshui, Hebei & Changchun, Jilin & Changping, Beijing & Qingdao, Shandong \\
\hline ZD958 & ZD958 & ZD958 & ZD958 & ZD958 \\
\hline XY335 & XY335 & XY335 & XY335 & XY335 \\
\hline XD20 & XD20 & XD20 & XD20 & XD20 \\
\hline XF32D22 & XF32D22 & XF32D22 & XF32D22 & XF32D22 \\
\hline LY13 & LY13 & LY13 & LY13 & LY13 \\
\hline XD18 & JH5 & NY309 & JH5 & JH5 \\
\hline YH988 & XQ73-1 & ND588 & XQ73-1 & LD981 \\
\hline HY14 & LY16 & PQ13 & LY16 & LD9032 \\
\hline YF335 & LD981 & SY103 & DH661 & LiaoYu22 \\
\hline XD29 & LY18 & DF77 & & LY35 \\
\hline ZK11 & LD9002 & XY508 & & LN14 \\
\hline \multirow[t]{2}{*}{ LD9 } & H311 & YQ281 & & $\mathrm{ZJ} 3$ \\
\hline & & JD26 & & \\
\hline
\end{tabular}


Table 2 Location, precipitation, and soil physicochemical characteristics at the maize study sites in China ${ }^{\text {a) }}$

\begin{tabular}{|c|c|c|c|c|c|c|c|c|c|c|c|c|c|c|c|}
\hline $\begin{array}{l}\text { Experiment } \\
\text { No. }\end{array}$ & Year & Location & $\begin{array}{l}\text { Latitude and } \\
\text { longitude }\end{array}$ & $\begin{array}{l}\text { Precipitation } \\
\text { during } \\
\text { growth } \\
\text { season }(\mathrm{mm}) \\
\end{array}$ & Soil type & \multicolumn{2}{|c|}{$\begin{array}{l}\mathrm{NaOH}-\mathrm{N} \\
\left(\mathrm{mg} \mathrm{kg}^{-1}\right)\end{array}$} & \multicolumn{2}{|c|}{$\begin{array}{l}\text { Olsen-P } \\
\left(\mathrm{mg} \mathrm{kg}^{-1}\right)\end{array}$} & \multicolumn{2}{|c|}{$\begin{array}{l}\mathrm{NH}_{4} \mathrm{Ac}-\mathrm{K} \\
\left(\mathrm{mg} \mathrm{kg}^{-1}\right)\end{array}$} & \multicolumn{2}{|c|}{$\begin{array}{l}\text { Organic } \\
\text { matter } \\
\left(\mathrm{g} \mathrm{kg}^{-1}\right)\end{array}$} & \multicolumn{2}{|c|}{$\mathrm{pH}$} \\
\hline 1 & 2008 & Xuchang, Henan & $34^{\circ} 07^{\prime} \mathrm{N}, 113^{\circ} 77^{\prime} \mathrm{E}$ & 397 & Alluvial soil & 5.23 & & 10.7 & & 80.0 & & 15.4 & & 7.90 & \\
\hline 2 & & Hengshui, Hebei & $37^{\circ} 45^{\prime} \mathrm{N}, 115^{\circ} 30^{\prime} \mathrm{E}$ & 400 & Alluvial soil & 15.6 & & 33.7 & & 106.4 & & 14.5 & & 8.45 & \\
\hline 3 & & Changchun, Jilin & $43^{\circ} 78^{\prime} \mathrm{N}, 125^{\circ} 38^{\prime} \mathrm{E}$ & 502 & Black soil & 5.9 & & 31.8 & & 110.1 & & 22.5 & & 6.67 & \\
\hline 5 & & $\begin{array}{l}\text { Qingdao, } \\
\text { Shandong }\end{array}$ & $36^{\circ} 38^{\prime} \mathrm{N}, 120^{\circ} 45^{\prime} \mathrm{E}$ & 501 & Brown soil & 20.2 & & 42.9 & & 94 & & 13.0 & & 5.69 & \\
\hline 6 & 2009 & $\begin{array}{l}\text { Qingdao, } \\
\text { Shandong }\end{array}$ & $36^{\circ} 38^{\prime} \mathrm{N}, 120^{\circ} 45^{\prime} \mathrm{E}$ & 416 & Brown soil & & & & & & & & & & \\
\hline 7 & & Changchun, Jilin & $43^{\circ} 78^{\prime} \mathrm{N}, 125^{\circ} 38^{\prime} \mathrm{E}$ & 367 & Black soil & & & & & & & & & & \\
\hline 8 & & $\begin{array}{c}\text { Changping, } \\
\text { Beijing }\end{array}$ & $40^{\circ} 09^{\prime} \mathrm{N}, 116^{\circ} 36^{\prime} \mathrm{E}$ & 360 & Alluvial soil & & & & & & & & & & \\
\hline
\end{tabular}

a) HN, high nitrogen; LN, low nitrogen. Data of precipitation were from the local meteorological bureaus. Experimental numbers are designated according to the experimental years.

$\mathrm{P}_{2} \mathrm{O}_{5} \mathrm{hm}^{-2}$ in all plots.

\subsection{Statistical analysis}

On the basis of the average yield under LN and HN, the tested cultivars were classified as either EE, HNE, LNE, or $\mathrm{NN}$. The potential of a cultivar to reduce $\mathrm{N}$ fertilizer input was estimated according to the method of Chen et al. [22] as the reduction in $\mathrm{N}$ fertilizer at which the yield of the cultivar equaled the average of all tested cultivars under HN. The calculations were as follows:

Agronomical $\mathrm{N}$ efficiency $(\mathrm{AE})=($ yield with $\mathrm{N}$ input -yield without $\mathrm{N}$ input)/ $\mathrm{N}$ input level,

Reduction in $\mathrm{N}$ fertilizer requirement $=\mathrm{N}$ inputlevel

-(average yield of all tested cultivars-yield of individual cultivar without $\mathrm{N}$ input)/AE of the cultivar,

Potential fertilizer reduction $(\%)=$ reduction in $\mathrm{N}$ fertilizer requirement/N input level $\times 100$,

Potential yield increase $(\%)=($ yield of a cultivar -average yield of all tested cultivars)/ average yield of all tested cultivars $\times 100$, NUE=yield/N input level [13],

Yield reduction at $\mathrm{LN}$ or $\mathrm{MN}(\%)=($ yield at $\mathrm{HN}$ -yield at $\mathrm{LN}$ or $\mathrm{MN}) /$ yield at $\mathrm{HN} \times 100$.

Heritability $\left(h^{2}\right)$ was estimated following Hallauer and Miranda [23]:

$$
h^{2} \%=\frac{\sigma_{\mathrm{g}}^{2}}{\sigma_{\mathrm{g}}^{2}+\sigma_{\mathrm{e}}^{2} / r} \times 100,
$$

where $\sigma_{\mathrm{g}}^{2}$ is the genetic variance, $\sigma_{\mathrm{e}}^{2}$ is the random error, and $r$ is the number of repeats.

Phenotypic correlation $\left(r_{\mathrm{p}}\right)$ and genetic correlation $\left(r_{\mathrm{g}}\right)$ were calculated as follows [24]:

$$
\begin{aligned}
& r_{\mathrm{p}}(x i x j)=\operatorname{COV}_{\mathrm{p}}(x i x j) / \sqrt{\delta_{\mathrm{p}}^{2}(x i) \delta_{\mathrm{p}}^{2}(x j)}, \\
& r_{\mathrm{g}}(x i x j)=\operatorname{COV}_{\mathrm{g}}(x i x j) / \sqrt{\delta_{\mathrm{g}}^{2}(x i) \delta_{\mathrm{g}}^{2}(x j)} .
\end{aligned}
$$

The experimental data were analyzed using two-way analysis of variance (ANOVA) with SAS software (SAS Institute, Cary, NC, USA) and differences were compared using the least significant difference (LSD) test at the 0.05 level of significance.

\section{Results}

\subsection{Variance analysis of yield}

Among the eight environments, the average heritability of yield was $75.8 \%$ and was unaffected by N-supply treatments (Table 3). The genotype $\times \mathrm{N}(\mathrm{G} \times \mathrm{N})$ interaction was significant in Experiments (Exps) 5-8, but not in Exps 1-4. The effect of $\mathrm{N}$ was also significant in Exps 5-8 (Table 4). In Exps 1-4, there was a significant correlation between yield at any two $\mathrm{N}$ levels. The only exception was in yield between $\mathrm{LN}$ and $\mathrm{HN}$ in Exp 4. In Exps 6-8, phenotypic and genetic correlations in yield were non-significant between $\mathrm{LN}$ and $\mathrm{HN}$ and between $\mathrm{LN}$ and MN.

\subsection{Variation in yield in response to $\mathbf{N}$ treatments in different environments}

In Exps $1-4$, in which the $\mathrm{G} \times \mathrm{N}$ interaction was not significant, LN slightly reduced yield by an average of $8.8 \%$ compared with that of HN (Table 5). The percentage reduction was highest at Xuchang, Henan (by 13.4\%) and lowest at Hengshui, Hebei (by 1.68\%). The coefficient of variation (7.98\%-8.74\%) was similar among the three $\mathrm{N}$ treatments. The yield under MN was not significantly different from that under HN, but NUE increased from $38.2 \mathrm{~kg} \mathrm{~kg}^{-1}$ under LN to $75.9 \mathrm{~kg} \mathrm{~kg}^{-1}$ under MN.

In Exps $5-8$, in which the $\mathrm{G} \times \mathrm{N}$ interaction was significant, the yield under LN was $39 \%$ lower than that under $\mathrm{HN}$, with a difference of $3788 \mathrm{~kg} \mathrm{hm}^{-2}$. The percentage reduc- 
Table 3 Heritability of yield and correlation with nitrogen (N) level in maize ${ }^{\text {a) }}$

\begin{tabular}{|c|c|c|c|c|c|c|c|c|c|}
\hline \multirow{2}{*}{ Experiment No. } & \multirow{2}{*}{$\begin{array}{c}\mathrm{LN} \\
h^{2}\end{array}$} & \multirow{2}{*}{$\begin{array}{c}\mathrm{MN} \\
h^{2}\end{array}$} & \multirow{2}{*}{$\begin{array}{c}\mathrm{HN} \\
h^{2}\end{array}$} & \multicolumn{2}{|c|}{ LN vs. MN } & \multicolumn{2}{|c|}{ LN vs. HN } & \multicolumn{2}{|c|}{ MN vs. $-\mathrm{HN}$} \\
\hline & & & & $r_{\mathrm{p}}$ & $r_{\mathrm{g}}$ & $r_{\mathrm{p}}$ & $r_{\mathrm{g}}$ & $r_{\mathrm{p}}$ & $r_{\mathrm{g}}$ \\
\hline 1 & 72.4 & 64.7 & 85.1 & $0.567^{*}$ & $0.801^{* *}$ & $0.664^{* *}$ & $0.843^{* *}$ & $0.558^{*}$ & $0.68^{* * *}$ \\
\hline 2 & 81.5 & 86.0 & 82.6 & $0.775^{* *}$ & $0.95^{* * * *}$ & $0.779^{* *}$ & $0.893^{* * * *}$ & $0.884^{* * * *}$ & $0.974^{* * * *}$ \\
\hline 3 & 37.1 & 63.6 & 50.7 & $0.812^{* * * *}$ & $1.07^{* * * * *}$ & $0.683^{* *}$ & $1.06^{* * * *}$ & $0.721^{* *}$ & $1.02^{* * * *}$ \\
\hline 4 & 56.9 & 84.2 & 69.1 & 0.615 & $0.891^{* * * *}$ & $0.757^{*}$ & $1.020^{* * * *}$ & $0.721^{*}$ & $0.979^{* * * *}$ \\
\hline 5 & 87.2 & 62.0 & 82.7 & $0.553^{*}$ & $0.814^{* *}$ & 0.489 & $0.573^{*}$ & $0.724^{* *}$ & $1.05^{* * * *}$ \\
\hline 6 & 86.2 & 87.7 & 77.3 & 0.041 & 0.0302 & -0.175 & -0.240 & $0.796^{* *}$ & $1.08^{* * * *}$ \\
\hline 7 & 81.4 & 74.4 & 86.2 & 0.163 & 0.196 & -0.309 & 0.160 & 0.365 & $1.01^{* * * *}$ \\
\hline 8 & 96.3 & 80.6 & 83.8 & 0.161 & 0.184 & -0.185 & -0.254 & $0.683^{*}$ & $0.731^{\text {** }}$ \\
\hline
\end{tabular}

a) Experiment numbers are described in Table 2 . LN, low-N treatment; MN, medium-N treatment; HN, high-N treatment; $h^{2}$, heritability; $r_{\mathrm{p}}$, phenotypic correlation; $r_{\mathrm{g}}$, genetic correlation. *, **, and *** indicate significance at $P<0.01, P<0.05$, and $P<0.001$ level, respectively.

Table 4 Analysis of variance in yield in the eight nitrogen $(\mathrm{N})$ experiments on maize ${ }^{\mathrm{a})}$

\begin{tabular}{|c|c|c|c|c|c|}
\hline Experiment No. & Block & $\mathrm{N}$ treatment & Genotype (G) & $\mathrm{G} \times \mathrm{N}$ & Error \\
\hline 1 & 1191934 & $14369263^{* * * *}$ & $4281028^{* * * *}$ & 814321 & 533120 \\
\hline 2 & $2450277^{* * * *}$ & 417789 & $4278164^{* * * *}$ & 341711 & 288733 \\
\hline 3 & 118756 & $17421263^{* * * *}$ & $3340509^{* * * *}$ & 354562 & 681735 \\
\hline 4 & 568841 & $13517231^{\text {**** }}$ & $4490705^{* * *}$ & 686626 & 526279 \\
\hline 5 & $8488851^{* * * *}$ & $176654000^{\text {*** }}$ & $5128346^{* * *}$ & $1008196^{*}$ & 617205 \\
\hline 6 & $2003330^{*}$ & $2003330^{\text {**** }}$ & $3380001^{* * * *}$ & $1754041^{* * * *}$ & 395034 \\
\hline 7 & 416202 & $137214683^{\text {**** }}$ & $3179078^{* * * *}$ & $3057113^{\text {**** }}$ & 692245 \\
\hline
\end{tabular}

a) Experiment numbers are described in Table 2. *, $P<0.05$; ***, $P<0.001$.

Table 5 Variation in yield in response to nitrogen $(\mathrm{N})$ treatments in eight fertilization experiments on maize ${ }^{\mathrm{a})}$

\begin{tabular}{|c|c|c|c|c|c|c|c|c|c|c|c|c|c|}
\hline \multirow[b]{2}{*}{$\begin{array}{c}\text { Experiment } \\
\text { No. }\end{array}$} & \multicolumn{4}{|c|}{$\mathrm{LN}$} & \multicolumn{5}{|c|}{$\mathrm{MN}$} & \multicolumn{4}{|c|}{$\mathrm{HN}$} \\
\hline & $\begin{array}{c}\text { Yield } \\
\left(\mathrm{kg} \mathrm{hm}^{-2}\right)\end{array}$ & $\begin{array}{c}\text { Variation } \\
\text { coefficient } \\
(\%)\end{array}$ & $\begin{array}{c}\text { Yield } \\
\text { reduction } \\
(\%)\end{array}$ & $\mathrm{LSD}_{0.05}$ & $\begin{array}{c}\text { Yield } \\
\left(\mathrm{kg} \mathrm{hm}^{-2}\right)\end{array}$ & $\begin{array}{c}\text { Variation } \\
\text { coeffi- } \\
\text { cient }(\%)\end{array}$ & $\begin{array}{l}\text { Yield } \\
\text { reduction } \\
(\%)\end{array}$ & $\begin{array}{c}\text { NUE } \\
\left(\mathrm{kg} \mathrm{kg}^{-1}\right)\end{array}$ & $\operatorname{LSD}_{0.05}$ & $\begin{array}{c}\text { Yield } \\
\left(\mathrm{kg} \mathrm{hm}^{-2}\right)\end{array}$ & $\begin{array}{c}\text { Variation } \\
\text { coefficient } \\
(\%)\end{array}$ & $\begin{array}{c}\mathrm{NUE} \\
\left(\mathrm{kg} \mathrm{kg}^{-1}\right)\end{array}$ & $\mathrm{LSD}_{0.05}$ \\
\hline \multicolumn{14}{|c|}{ Experiments in which $\mathrm{G} \times \mathrm{N}$ interaction was non-significant } \\
\hline 1 & 7260 & 10.1 & 13.4 & 1354 & 8101 & 9.18 & 3.38 & 67.5 & 1281 & 8384 & 11.2 & 34.9 & 1064 \\
\hline 2 & 8260 & 8.90 & 1.68 & 916 & 8453 & 10.0 & -0.61 & 70.4 & 1186 & 8402 & 7.45 & 35.0 & 780 \\
\hline 3 & 9181 & 7.04 & 11.7 & 1479 & 10034 & 6.77 & 3.45 & 83.6 & 1205 & 10393 & 6.61 & 43.3 & 1392 \\
\hline 4 & 9752 & 5.90 & 8.55 & 1239 & 11061 & 9.02 & -3.73 & 81.9 & 1170 & 10663 & 7.43 & 39.5 & 1299 \\
\hline Mean & 8613 & 7.98 & 8.82 & & 9412 & 8.74 & 0.62 & 75.9 & & 9460 & 8.17 & 38.2 & \\
\hline SE & 546 & 0.94 & 2.58 & & 692 & 0.69 & 1.73 & 4.04 & & 619 & 1.03 & 2.01 & \\
\hline \multicolumn{14}{|c|}{ Experiments in which $\mathrm{G} \times \mathrm{N}$ interaction was significant } \\
\hline 5 & 4906 & 19.0 & 42.5 & 1395 & 8371 & 9.31 & 1.96 & 69.8 & 1628 & 8538 & 11.2 & 35.6 & 1469 \\
\hline 6 & 4198 & 20.0 & 56.1 & 1053 & 8924 & 10.7 & 6.61 & 74.4 & 1109 & 9556 & 8.63 & 39.8 & 1139 \\
\hline 7 & 8198 & 14.4 & 27.0 & 1717 & 11212 & 6.59 & 0.18 & 93.4 & 1162 & 11233 & 9.62 & 46.8 & 1193 \\
\hline 8 & 7200 & 13.3 & 30.3 & 661 & 10076 & 9.08 & 2.43 & 74.6 & 1187 & 10327 & 9.35 & 38.2 & 1161 \\
\hline Mean & 6126 & $16.7^{* * *}$ & 39.0 & & 9646 & 8.92 & 2.80 & 78.0 & & 9914 & 9.70 & 40.1 & \\
\hline SE & 942 & 1.66 & 6.60 & & 631 & 0.86 & 1.36 & 5.25 & & 572 & 0.54 & 2.40 & \\
\hline
\end{tabular}

a) LN, low-N treatment; MN, medium-N treatment; HN, high-N treatment; LSD, least significant difference; NUE, nitrogen use efficiency; G $\times N$, genotypexnitrogen treatment interaction; SE, standard error. ${ }^{* *}, P<0.01$ by analysis of variance.

tion was highest at Qingdao, Shandong (by $42.5 \%$ and $56.1 \%$, in 2008 and 2009 , respectively). The variation coefficients for $\mathrm{LN}$ and $\mathrm{MN}$ were significantly higher than for $\mathrm{HN}$, suggesting large differences in the responses to $\mathrm{N}$ treatment among cultivars. The yield under MN was not significantly different from that under HN, but NUE increased from $40.1 \mathrm{~kg} \mathrm{~kg}^{-1}$ under LN to $78 \mathrm{~kg} \mathrm{~kg}^{-1}$ under MN.

\subsection{Potential fertilizer reduction and yield increase by $\mathrm{N}$-efficient cultivars}

On the basis of average yield in the LN and HN treatments, the cultivars were classified into four NUE classes using the data obtained in Exps 5-8. For each NUE class, the average yield, percentage reduction, NUE, reduction in $\mathrm{N}$ fertilizer requirement, potential fertilizer input reduction, and potential yield increase were calculated (Tables 6-9).

Fifteen cultivars $(28 \%$ of the total cultivar numbers used in Exps 5-8) were classified as LNE (Table 6). Under LN, the yield of LNE cultivars was $11.8 \%$ higher than the average yield of all tested cultivars and average yield reduction was $26.3 \%$. Under MN and HN, the yields of LNE cultivars were $3.25 \%$ and $7.76 \%$ lower, respectively, than the average yield of all tested cultivars. No potential $\mathrm{N}$ savings were 
observed for this class of cultivars. The yield performance of LNE cultivars was variable, with only XD20 and ND108 showing the same performance.

Ten cultivars (19\% of the total cultivar numbers used in Exps 5-8) were classified as HNE (Table 7). Under LN, the yield of HNE cultivars was $15.4 \%$ lower than the average yield of all tested cultivars, and under $\mathrm{HN}$, it was $9.46 \%$ higher. The average yield reduction was $52.6 \%$. The potential $\mathrm{N}$ fertilizer savings was $20.7 \%$. XY335 was a typical HNE cultivar in which yield performance was identical in.

Thirteen cultivars $(25 \%$ of the total cultivar numbers used in Exps 5-8) were classified as EE (Table 8). Under $\mathrm{LN}, \mathrm{MN}$, and $\mathrm{HN}$, the yields of EE cultivars were $15 \%$, $6.62 \%$, and $7.57 \%$ higher, respectively, than the average yields of all tested cultivars. The average yield reduction was $34.7 \%$. The potential $\mathrm{N}$ fertilizer savings was $25.2 \%-$ $15.9 \%$. ZD958 was a typical HNE cultivar in which yield performance was identical.

Fifteen cultivars $(28 \%$ of the total cultivar numbers used in Exps 5-8) were classified as NN (Table 9). Under LN, $\mathrm{MN}$, and $\mathrm{HN}$, the yields of NN cultivars were $13.5 \%, 4.26 \%$, and $4.74 \%$ lower than the average yield of all tested cultivars. The average yield reduction was $44.6 \%$. No potential $\mathrm{N}$ fertilizer savings was observed for this class of cultivars.

The yields of cultivars within a NUE class were averaged and compared for each $\mathrm{N}$ treatment (Figure 1). The yield of
EE cultivars was significantly higher than that of HNE cultivars under LN but not under MN and HN. Thus, HNE cultivars (such as XY335) were more sensitive to low $\mathrm{N}$ than were EE cultivars (such as ZD958). The yield of LNE cultivars (such as ND108 and XD20) was significantly higher than that of NN cultivars under LN but not under HN and MN. For most of the tested cultivars, the NUE varied among environments.

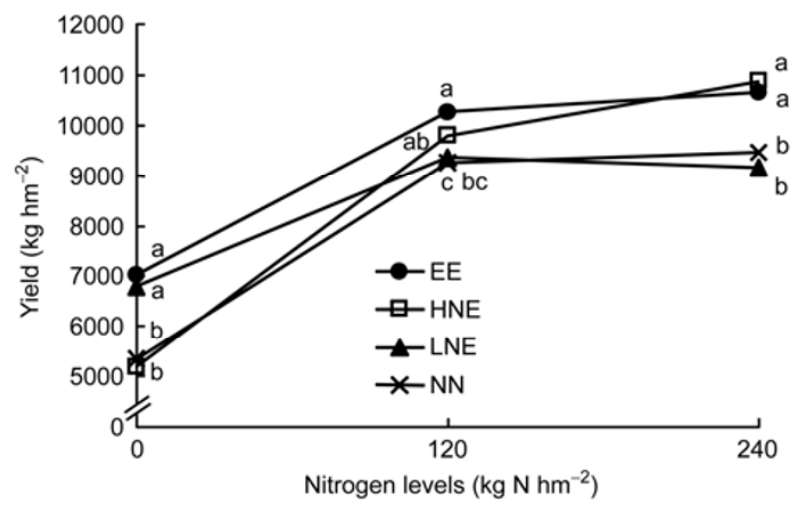

Figure 1 Yield of cultivars in each nitrogen use efficiency (NUE) class in response to three $\mathrm{N}$ application levels. Data are derived from Tables 6-9. The yield of the cultivars in each NUE class was averaged and compared for each $\mathrm{N}$ treatment. Points at the same nitrogen level with different lowercase letters were significantly different at the $P<0.05$ level by the LSD test.

Table 6 Yield and nitrogen $(\mathrm{N})$ responsiveness of low-nitrogen-efficient cultivars ${ }^{\text {a) }}$

\begin{tabular}{|c|c|c|c|c|c|c|c|}
\hline $\mathrm{N}$ treatment & Parameter & $\begin{array}{l}\text { Experiment } \\
\text { No. } 5\end{array}$ & $\begin{array}{c}\text { Experiment } \\
\text { No. } 6\end{array}$ & $\begin{array}{c}\text { Experiment } \\
\text { No. } 7\end{array}$ & $\begin{array}{c}\text { Experiment } \\
\text { No. } 8\end{array}$ & Mean & SE \\
\hline \multirow{5}{*}{$\mathrm{LN}$} & Cultivars & $\begin{array}{l}\text { XD20, LY22, } \\
\text { LN14 }\end{array}$ & $\begin{array}{c}\text { ND108, } \\
\text { LD9032, } \\
\text { LD981, ZJ3 }\end{array}$ & $\begin{array}{l}\text { ND588, JD26, } \\
\text { XD20, YF29, } \\
\text { YQ281 }\end{array}$ & $\begin{array}{c}\text { LY13, } \\
\text { DH3719, } \\
\text { ND108 }\end{array}$ & & \\
\hline & Average yield $\left(\mathrm{kg} \mathrm{hm}^{-2}\right)$ & 5662 & 4881 & 8858 & 7731 & 6783 & 917 \\
\hline & Yield reduction compared with HN (\%) & 27.6 & 46.2 & 15.2 & 16.3 & 26.3 & 7.20 \\
\hline & $\begin{array}{l}\text { Yield increase compared with the average } \\
\text { yield of all tested cultivars }\left(\mathrm{kg} \mathrm{hm}^{-2}\right)\end{array}$ & 756 & 683 & 660 & 531 & 657 & 46.8 \\
\hline & Percentage yield increase $(\%)$ & 15.4 & 16.3 & 8.06 & 7.38 & 11.8 & 2.35 \\
\hline \multirow{6}{*}{ MN } & Average yield $\left(\mathrm{kg} \mathrm{hm}^{-2}\right)$ & 8081 & 8647 & 11076 & 9548 & 9338 & 653 \\
\hline & Yield reduction compared with $\mathrm{HN}(\%)$ & -3.27 & 4.67 & -6.05 & -3.42 & -2.02 & 2.32 \\
\hline & Average NUE $\left(\mathrm{kg} \mathrm{kg}^{-1}\right)$ & 67.3 & 72.1 & 92.3 & 79.6 & 77.8 & 5.44 \\
\hline & $\begin{array}{l}\text { Reduction in } \mathrm{N} \text { fertilizer requirement } \\
\left(\mathrm{kg} \mathrm{hm}^{-2}\right)\end{array}$ & -10.9 & -7.47 & -5.67 & -30.3 & -13.6 & 5.69 \\
\hline & Potential $\mathrm{N}$ fertilizer input reduction (\%) & -9.12 & -6.22 & -4.72 & -22.5 & -10.6 & 4.05 \\
\hline & Percentage yield increase $(\%)$ & -3.46 & -3.10 & -1.21 & -5.24 & -3.25 & 0.83 \\
\hline \multirow{6}{*}{$\mathrm{HN}$} & Average yield $\left(\mathrm{kg} \mathrm{hm}^{-2}\right)$ & 7825 & 9071 & 10444 & 9233 & 9143 & 536 \\
\hline & Average NUE $\left(\mathrm{kg} \mathrm{kg}^{-1}\right)$ & 32.6 & 37.8 & 43.5 & 34.2 & 37.0 & 2.42 \\
\hline & $\begin{array}{l}\text { Reduction in } \mathrm{N} \text { fertilizer requirement } \\
\left(\mathrm{kg} \mathrm{hm}^{-2}\right)\end{array}$ & -58.6 & -23.9 & -84.3 & -145 & -78.0 & 25.6 \\
\hline & Potential N fertilizer input reduction (\%) & -24.4 & -9.95 & -35.1 & -53.8 & -30.8 & 9.24 \\
\hline & $\begin{array}{l}\text { Yield increase compared with the average } \\
\text { yield of all tested cultivars }\left(\mathrm{kg} \mathrm{hm}^{-2}\right)\end{array}$ & -713 & -485 & -789 & -1094 & -770 & 126 \\
\hline & Percentage yield increase $(\%)$ & -8.35 & -5.07 & -7.02 & -10.6 & -7.76 & 1.16 \\
\hline
\end{tabular}

a) LN, low-N treatment; MN, medium-N treatment; HN, high-N treatment; SE, standard error. 
Table 7 Yield and nitrogen $(\mathrm{N})$ responsiveness of high-nitrogen-efficient cultivars ${ }^{\text {a) }}$

\begin{tabular}{|c|c|c|c|c|c|c|c|}
\hline $\mathrm{N}$ treatment & Parameter & $\begin{array}{l}\text { Experiment } \\
\quad \text { No. } 5\end{array}$ & $\begin{array}{l}\text { Experiment } \\
\text { No. } 6\end{array}$ & $\begin{array}{l}\text { Experiment } \\
\text { No. } 7 \\
\end{array}$ & $\begin{array}{l}\text { Experiment } \\
\text { No. } 8 \\
\end{array}$ & Mean & SE \\
\hline \multirow{5}{*}{$\mathrm{LN}$} & Cultivars & $\begin{array}{l}\text { LD9032, } \\
\text { XY3355 }\end{array}$ & $\begin{array}{c}\text { LY13, } \\
\text { XF32D22, } \\
\text { XY335 }\end{array}$ & $\begin{array}{l}\text { DF77, XY335, } \\
\quad \text { XY508 }\end{array}$ & XY335, JH5 & & \\
\hline & Average yield $\left(\mathrm{kg} \mathrm{hm}^{-2}\right)$ & 4550 & 3183 & 6737 & 6309 & 5195 & 821 \\
\hline & Yield reduction compared with HN (\%) & 50.8 & 69.5 & 46.3 & 43.9 & 52.6 & 5.79 \\
\hline & $\begin{array}{l}\text { Yield increase compared with the aver- } \\
\text { age yield of all tested cultivars }\left(\mathrm{kg} \mathrm{hm}^{-2}\right)\end{array}$ & -356 & -1015 & -1461 & -891 & -931 & 227 \\
\hline & Percentage yield increase $(\%)$ & -7.25 & -24.2 & -17.8 & -12.4 & -15.4 & 3.63 \\
\hline \multirow{7}{*}{ MN } & Average yield $\left(\mathrm{kg} \mathrm{hm}^{-2}\right)$ & 8143 & 9449.6 & 11085 & 10475 & 9788 & 644 \\
\hline & Yield reduction compared with $\mathrm{HN}(\%)$ & 11.9 & 9.32 & 11.6 & 6.88 & 9.92 & 1.16 \\
\hline & Average NUE $\left(\mathrm{kg} \mathrm{kg}^{-1}\right)$ & 67.9 & 78.7 & 92.4 & 77.6 & 79.1 & 5.04 \\
\hline & $\begin{array}{l}\text { Reduction in } \mathrm{N} \text { fertilizer requirement } \\
\left(\mathrm{kg} \mathrm{hm}^{-2}\right)\end{array}$ & -8.45 & 12.0 & -5.26 & 16.5 & 3.69 & 6.19 \\
\hline & Potential $\mathrm{N}$ fertilizer input reduction (\%) & -7.04 & 10.0 & -4.39 & 12.2 & 2.69 & 4.90 \\
\hline & $\begin{array}{l}\text { Yield increase compared with the aver- } \\
\text { age yield of all tested cultivars }\left(\mathrm{kg} \mathrm{hm}^{-2}\right)\end{array}$ & -228 & 526 & -127 & 399 & 143 & 188 \\
\hline & Percentage yield increase $(\%)$ & -2.72 & 5.89 & -1.13 & 3.96 & 1.50 & 2.04 \\
\hline \multirow{6}{*}{$\mathrm{HN}$} & Average yield $\left(\mathrm{kg} \mathrm{hm}^{-2}\right)$ & 9243 & 10421 & 12537 & 11249 & 10863 & 694 \\
\hline & Average NUE $\left(\mathrm{kg} \mathrm{kg}^{-1}\right)$ & 38.5 & 43.4 & 52.2 & 41.7 & 44.0 & 2.94 \\
\hline & $\begin{array}{l}\text { Reduction in } \mathrm{N} \text { fertilizer requirement } \\
\left(\mathrm{kg} \mathrm{hm}^{-2}\right)\end{array}$ & 39.0 & 33.4 & 72.1 & 61.5 & 51.5 & 9.18 \\
\hline & Potential $\mathrm{N}$ fertilizer input reduction $(\%)$ & 16.3 & 13.9 & 30.1 & 22.8 & 20.7 & 3.63 \\
\hline & $\begin{array}{l}\text { Yield increase compared with the aver- } \\
\text { age yield of all tested cultivars }\left(\mathrm{kg} \mathrm{hm}^{-2}\right)\end{array}$ & 705 & 865 & 1304 & 922 & 949 & 127 \\
\hline & Percentage yield increase $(\%)$ & 8.25 & 9.05 & 11.6 & 8.93 & 9.46 & 0.74 \\
\hline
\end{tabular}

a) LN, low-N treatment; $\mathrm{MN}$, medium-N treatment; HN, high-N treatment; SE, standard error.

Table 8 Yield and nitrogen (N) responsiveness of efficient-efficient cultivars ${ }^{\text {a) }}$

\begin{tabular}{|c|c|c|c|c|c|c|c|}
\hline $\mathrm{N}$ treatment & Parameter & $\begin{array}{l}\text { Experiment } \\
\text { No. } 5 \\
\end{array}$ & $\begin{array}{l}\text { Experiment } \\
\text { No. } 6 \\
\end{array}$ & $\begin{array}{l}\text { Experiment } \\
\text { No. } 7\end{array}$ & $\begin{array}{l}\text { Experiment } \\
\text { No. } 8 \\
\end{array}$ & Mean & SE \\
\hline \multirow{5}{*}{$\mathrm{LN}$} & Cultivars & $\begin{array}{l}\text { ZD958, LY13, } \\
\text { LY35 }\end{array}$ & $\begin{array}{l}\text { XD20, ZD958, } \\
\text { LY35, QD1 }\end{array}$ & $\begin{array}{l}\text { PQ13, JD137, } \\
\text { XF32D22 }\end{array}$ & $\begin{array}{c}\text { XF32D22, } \\
\text { XQ73-1, ZD958 }\end{array}$ & & \\
\hline & Average yield $\left(\mathrm{kg} \mathrm{hm}^{-2}\right)$ & 6007 & 4742 & 9266 & 8038 & 7013 & 1012 \\
\hline & Yield reduction compared with $\mathrm{HN}(\%)$ & 39.4 & 52.6 & 21.2 & 25.7 & 34.7 & 7.12 \\
\hline & $\begin{array}{l}\text { Yield increase compared with the average } \\
\text { yield of all tested cultivars }\left(\mathrm{kg} \mathrm{hm}^{-2}\right)\end{array}$ & 1101 & 544 & 1068 & 838 & 888 & 129 \\
\hline & Percentage yield increase $(\%)$ & 22.4 & 13.0 & 13.0 & 11.6 & 15.0 & 2.50 \\
\hline \multirow{7}{*}{ MN } & Average yield $\left(\mathrm{kg} \mathrm{hm}^{-2}\right)$ & 9287 & 9443 & 11749 & 10572 & 10263 & 572 \\
\hline & Yield reduction compared with $\mathrm{HN}(\%)$ & 6.32 & 5.67 & 0.03 & 2.32 & 3.59 & 1.47 \\
\hline & Average NUE $\left(\mathrm{kg} \mathrm{kg}^{-1}\right)$ & 77.4 & 78.7 & 97.9 & 78.3 & 83.1 & 4.95 \\
\hline & $\begin{array}{l}\text { Reduction in } \mathrm{N} \text { fertilizer requirement } \\
\left(\mathrm{kg} \mathrm{hm}^{-2}\right)\end{array}$ & 25.1 & 11.9 & 18.2 & 19.8 & 18.7 & 2.72 \\
\hline & Potential $\mathrm{N}$ fertilizer input reduction (\%) & 20.9 & 9.89 & 15.1 & 14.7 & 15.2 & 2.25 \\
\hline & $\begin{array}{l}\text { Yield increase compared with the average } \\
\text { yield of all tested cultivars }\left(\mathrm{kg} \mathrm{hm}^{-2}\right)\end{array}$ & 916 & 519 & 537 & 496 & 617 & 100 \\
\hline & Percentage yield increase $(\%)$ & 10.9 & 5.81 & 4.79 & 4.92 & 6.62 & 1.46 \\
\hline \multirow{6}{*}{$\mathrm{HN}$} & Average yield $\left(\mathrm{kg} \mathrm{hm}^{-2}\right)$ & 9913 & 10011 & 11752 & 10823 & 10625 & 428 \\
\hline & Average NUE $\left(\mathrm{kg} \mathrm{kg}^{-1}\right)$ & 41.3 & 41.7 & 49.0 & 40.1 & 43.0 & 2.01 \\
\hline & $\begin{array}{l}\text { Reduction in } \mathrm{N} \text { fertilizer requirement } \\
\left(\mathrm{kg} \mathrm{hm}^{-2}\right)\end{array}$ & 65.9 & 18.8 & 35.1 & 37.0 & 39.2 & 9.80 \\
\hline & Potential $\mathrm{N}$ fertilizer input reduction $(\%)$ & 27.5 & 7.82 & 14.6 & 13.7 & 15.9 & 4.14 \\
\hline & $\begin{array}{l}\text { Yield increase compared with the average } \\
\text { yield of all tested cultivars }\left(\mathrm{kg} \mathrm{hm}^{-2}\right)\end{array}$ & 1375 & 455 & 519 & 496 & 711 & 222 \\
\hline & Percentage yield increase $(\%)$ & 16.1 & 4.76 & 4.62 & 4.80 & 7.57 & 2.85 \\
\hline
\end{tabular}

a) LN, low-N treatment; MN, medium-N treatment; HN, high-N treatment; SE, standard error. 
Table 9 Yield and nitrogen $(\mathrm{N})$ responsiveness of nonefficient-nonefficient cultivars ${ }^{\mathrm{a})}$

\begin{tabular}{|c|c|c|c|c|c|c|c|}
\hline $\mathrm{N}$ treatment & Parameter & $\begin{array}{l}\text { Experiment } \\
\text { No. } 5 \\
\end{array}$ & $\begin{array}{l}\text { Experiment } \\
\text { No. } 6\end{array}$ & $\begin{array}{l}\text { Experiment } \\
\text { No. } 7\end{array}$ & $\begin{array}{l}\text { Experiment } \\
\text { No. } 8 \\
\end{array}$ & Mean & SE \\
\hline \multirow{5}{*}{$\mathrm{LN}$} & Cultivars & $\begin{array}{c}\text { XF1, ZJ3, JH5, } \\
\text { XF32D22, } \\
\text { ND108, LD981 }\end{array}$ & $\begin{array}{l}\text { JH5, LN14, } \\
\text { LiaoY22 }\end{array}$ & $\begin{array}{l}\text { ZD958, SY103, } \\
\text { NY309, LY13 }\end{array}$ & XD20, LY16 & & \\
\hline & Average yield $\left(\mathrm{kg} \mathrm{hm}^{-2}\right)$ & 4096 & 3578 & 7666 & 6039 & 5345 & 938 \\
\hline & Yield reduction compared with HN (\%) & 48.6 & 59.0 & 29.3 & 41.4 & 44.6 & 6.24 \\
\hline & $\begin{array}{l}\text { Yield increase compared with the aver- } \\
\text { age yield of all tested cultivars }\left(\mathrm{kg} \mathrm{hm}^{-2}\right)\end{array}$ & -810 & -620 & -532 & -1161 & -781 & 140 \\
\hline & Percentage yield increase $(\%)$ & -16.5 & -14.8 & -6.48 & -16.1 & -13.5 & 2.36 \\
\hline \multirow{6}{*}{$\mathrm{MN}$} & Average yield $\left(\mathrm{kg} \mathrm{hm}^{-2}\right)$ & 8135 & 8076 & 11074 & 9725 & 9253 & 717 \\
\hline & Yield reduction compared with $\mathrm{HN}(\%)$ & -2.03 & 7.48 & -2.07 & 5.59 & 2.24 & 2.51 \\
\hline & Average NUE $\left(\mathrm{kg} \mathrm{kg}^{-1}\right)$ & 121 & 112 & 120 & 122 & 119 & 2.28 \\
\hline & $\begin{array}{l}\text { Reduction in } \mathrm{N} \text { fertilizer requirement } \\
\left(\mathrm{kg} \mathrm{hm}^{-2}\right)\end{array}$ & -8.77 & -26.2 & -5.75 & -18.8 & -14.9 & 4.70 \\
\hline & Potential $\mathrm{N}$ fertilizer input reduction (\%) & -7.31 & -21.9 & -4.79 & -13.9 & -12.0 & 3.82 \\
\hline & Percentage yield increase $(\%)$ & -2.82 & -9.50 & -1.23 & -3.49 & -4.26 & 1.81 \\
\hline \multirow{6}{*}{$\mathrm{HN}$} & Average yield $\left(\mathrm{kg} \mathrm{hm}^{-2}\right)$ & 7973 & 8729 & 10850 & 10300 & 9463 & 670 \\
\hline & Average NUE $\left(\mathrm{kg} \mathrm{kg}^{-1}\right)$ & 33.2 & 36.4 & 45.2 & 38.1 & 38.2 & 2.54 \\
\hline & $\begin{array}{l}\text { Reduction in } \mathrm{N} \text { fertilizer requirement } \\
\left(\mathrm{kg} \mathrm{hm}^{-2}\right)\end{array}$ & -44.2 & -43.8 & -34.7 & -2.36 & -31.3 & 9.88 \\
\hline & Potential $\mathrm{N}$ fertilizer input reduction (\%) & -18.4 & -18.2 & -14.5 & -0.87 & -13.0 & 4.14 \\
\hline & $\begin{array}{l}\text { Yield increase compared with the aver- } \\
\text { age yield of all tested cultivars }\left(\mathrm{kg} \mathrm{hm}^{-2}\right)\end{array}$ & -565 & -827 & -383 & -27.1 & -451 & 168 \\
\hline & Percentage yield increase $(\%)$ & -6.62 & -8.65 & -3.41 & -0.26 & -4.74 & 1.84 \\
\hline
\end{tabular}

a) LN, low-N treatment; MN, medium-N treatment; HN, high-N treatment; SE, standard error.

\section{Discussion}

\subsection{Genotypexnitrogen interaction and the pressure for NUE selection}

Bänzinger et al. [19] suggested that selection of N-efficient genotypes should (i) include adequate sources of genetic variation and strong selection pressures for the important traits at all stages of the breeding program, (ii) use experimental procedures to achieve high levels of heritability in the breeding trials, and (iii) employ tests that achieve a high genetic correlation between germplasm performance in breeding trials and under farm conditions. Yield in the field is the most important nutrient efficiency parameter. Soil $\mathrm{N}$ availability is the key selection pressure that determines selection efficiency. In the present study, coefficients of variation were significantly different among $\mathrm{N}$ treatments only in the experiments in which the $\mathrm{G} \times \mathrm{N}$ interaction was significant. Therefore, only under sufficiently low-N stress could the maximum genetic variation be observed. According to studies by CIMMYT, selection for LN tolerance should be conducted in fields in which LN yields are $25 \%-35 \%$ of those in N-sufficient plots. However, others have suggested that yield in LN plots should be $60 \%-65 \%$ of those in N-sufficient plot [25], in which case the $\mathrm{N}$ level may be too high for selection of LNE-type genotypes [15]. The present results suggested that when the yield of LN plots was $25 \%-60 \%$ of that of $\mathrm{HN}$ plots, the $\mathrm{G} \times \mathrm{N}$ interac- tion on yield was significant and yield heritability remained about $75 \%$. Under LN stress, the variation in yield among genotypes was sufficiently high to classify them into different NUE classes. If yield reduction is less than $15 \%$, a significant $\mathrm{G} \times \mathrm{N}$ interaction will be difficult to obtain.

A correlation between yields under $\mathrm{HN}$ and $\mathrm{LN}$ is frequently observed. If LN stress in the target area is not serious (yield reduction is less than $10 \%$, for example), selection for high yield in multiple environments can also increase yield performance under LN [26]. Nevertheless, with increasing LN stress, the correlation between yield under $\mathrm{HN}$ and LN decreases [27,28]. In the present study, when yield reduction was as high as $27.0 \%-56.1 \%$, the correlation between the yields under $\mathrm{HN}$ and $\mathrm{LN}$ was no longer significant (Table 5). To obtain LNE and EE genotypes, selection in both LN and HN environments is necessary [15]. Selection in a LN environment can increase selection efficiency by $30 \%$. If the correlation between yields under $\mathrm{LN}$ and $\mathrm{HN}$ is about $0.65, \mathrm{LN}$-tolerant genotypes selected in LN environments often achieve a higher yield under $\mathrm{HN}$ [28]. Therefore, breeding for NUE in maize should be conducted in both low and high $\mathrm{N}$ environment and the yield reduction under low $\mathrm{N}$ plots should be $25 \%$ to $60 \%$.

\subsection{Potential reduction in $\mathbf{N}$ fertilizer input and/or in- creased yield with $\mathrm{N}$-efficient cultivars}

The standard for NUE classification depends on the aim of 
the study. With reference to Liu et al. [20] and Mi et al. [18], the cultivars tested in the present study were classified as HNE, LNE, EE, or NN. By comparing each yield performance with the average yield of all tested cultivars, the potential reduction in $\mathrm{N}$ fertilizer input and/or increased yield via use of $\mathrm{N}$-efficient cultivars were estimated. The current commercially grown HNE and EE cultivars have the potential to achieve an increased yield of $8 \%-10 \%$ and/or reduce $\mathrm{N}$ fertilizer input by $16 \%-21 \%$. The HNE cultivars were highly responsive to $\mathrm{N}$ fertilizer, but suffered a high loss in yield (by $52.6 \%$ ) under LN stress. These cultivars can only achieve high yields under HN input [29]. The EE cultivars achieve high yields under both LN and HN conditions and should be used for breeding in the future. Both HNE (such as XY335) and EE cultivars (such as ZD958) should be treated as $\mathrm{N}$-efficient cultivars in an intensive cropping system (Tables 7 and 8). The present results are compatible with those of previous studies. Worku et al. [30] reported that EE cultivars had the potential for a $10.7 \%$ increase in yield and $12.7 \%$ reduction in $\mathrm{N}$ fertilizer input. In contrast, HNE cultivars could achieve $15.1 \%$ increased yield and $17 \%$ reduction in $\mathrm{N}$ fertilizer input. In France, Coque and Gallais [31] reported that variation in the yield of current commercial cultivars was small. However, the results of the present study suggested that, in North and Northeast China, maize yield can be increased by $10 \%-15 \%$ and $\mathrm{N}$ fertilizer input could be reduced by $10 \%-20 \%$ if EE and/or HNE cultivars were used.

Nitrogen deficiency is a worldwide problem in crop production. In India, 2.5 million $\mathrm{hm}^{2}$ of arable land suffers from $\mathrm{N}$ deficiency, which results in a 50\% reduction in yield [32]. In South China, the area of N-deficient land is 1.15 million $\mathrm{hm}^{2}$, which results in a yield reduction of $10 \%-20 \%$. In Brazil, more than $80 \%$ of arable land has low fertility, and maize yield is only 1-2 tons $\mathrm{hm}^{-2}$ [33]. The maize yield in Africa is only 1.3 tons $\mathrm{hm}^{-2}$ because of LN and drought stress. Therefore, LNE cultivars are crucial to address the food security problem worldwide [15,34]. In the present study, LNE cultivars showed a potential increase in yield of 12\% (Table 7). XD20 and ND108 were typical LNE cultivars and were LN tolerant. ND108 was found to be LN tolerant in previous studies [35,36]. LNE cultivars bred by CIMMYT have shown excellent performance in Africa, producing yields of $2-5$ ton $\mathrm{hm}^{-2}$, which is $11 \%-$ $20 \%$ higher than the local cultivars [34]. At Hohenheim University, Germany, cultivars developed under LN stress produce $12 \%$ higher yield than those developed under HN conditions when grown in a LN environment [37]. Worku et al. [30] reported that the potential to increase yield of LNE cultivars was $14.5 \%$. Collectively, these studies indicate that LNE cultivars may increase maize yield by $10 \%-20 \%$.

This work was supported by the National Basic Research Program of China (2011CB100305, 2009CB11860), the National Natural Science Foun- dation of China (31121062, 31172015), and the Special Fund for Agriculture Profession (201103003).

1 Maplecroft. Food Security Risk Index (FSRI). 2011. http:// maplecroft.com/about/news/food_security.html

2 The National Development and Reform Commission. The National Food Security and Long-term Planning Framework (2008-2020) (in Chinese). The People's Republic of China. http://www.gov.cn

3 Zhang F S, Cui Z L, Wang J Q, et al. Current status of soil and plant nutrient management in China and improvement strategies (in Chinese). Chin Bull Bot, 2007, 24: 687-694

4 Matson P A, Parton W J, Power A G, et al. Agricultural intensification and ecosystem properties. Science, 1997, 277: 504-509

5 Tilman D, Cassman K G, Matson P A, et al. Agricultural sustainability and intensive production practices. Nature, 2002, 418: 671-678

6 Zhang F S, Mi G H, Liu J A. Advances in the genetic improvement of nitrogen efficiency in maize (in Chinese). J Agric Biotech, 1997, 5: 112-117

7 Li C H, Su X H, Xie R Z, et al. Study on relationship between grain-yield of summer corn and climatic ecological condition under super-high-yield cultivation (in Chinese). Sci Agric Sinica, 2001, 34: 311-316

8 Li D H, Zhang Y H, Yang J S, et al. The breeding and cultivation of combining compact type maize of high yield (in Chinese). J Maize Sci, 2004, 12: 69-71

9 Chen G P, Zhao J R, Zhang J W, et al. Study on the record of spring maize cultivation technology (in Chinese). J Maize Sci, 1995, 3: 26-30

10 Chen X P, Cui Z L, Vitousekb P M, et al. Integrated soil-crop system management for food security. Proc Natl Acad Sci USA, 2011, 108: 6399-6404

11 Food and Agriculture Organization of the United Nations. The agricultural production domain covers. 1995. http://faostat.fao.org/site/ 339/default.aspx

12 Pollmer W G, Eberhard D, Klein D, et al. Studies on maize hybrids involving inbred lines with varying protein content. Z Pflanzenzuecht, 1978, 80: 142-148

13 Moll R H, Kamprath E J, Jackson W A. Analysis and interpretation of factors which contribute to efficiency of nitrogen utilization. Agron J, 1982, 74: 562-568

14 Mi G H, Liu J A, Zhang F S. Analysis on agronomic nitrogen efficiency and its components of maize hybrids (in Chinese). J China Agric Univ, 1998, 3: 97-104

15 Bänzinger M, Edmeades G O, Beck D, et al. Breeding for Drought and Nitrogen Stress Tolerance in Maize: from Theory to Practice. Mexico: International Maize and Wheat Improvement Center, 2000

16 Luce G, Mathesius J. Hybrid response to nitrogen fertilizer: are there differences? Pioneer Agron Sci, 2009, 19: 1-3

17 Chen F J, Mi G H, Zhang F S. Breeding of nitrogen use efficiency on maize cultivar Zhongnong 99 (in Chinese). Crops, 2009, 6: 103-104

18 Mi G H, Chen F J, Zhang F S. Physiological Basis and Genetic Improvement of Crop Nutrient Efficiency (in Chinese). Beijing: China Agricultural University Press, 2012. 1-73

19 Bänzinger M, Cooper M. Breeding for low input conditions and consequences for participatory plant breeding: examples from tropical maize and wheat. Euphytica, 2001, 122: 503-519

20 Liu J A, Mi G H, Zhang F S. Difference in nitrogen efficiency among maize genotypes (in Chinese). J Agric Biotech, 1999, 7: 248-254

21 Guo L P, Wang X R, Zhang F S, et al. Effect of fertilizer application in different years on crop yields and fertilizer recovery (in Chinese). Chinese J Agrometeor, 1999, 20: 20-23

22 Chen F J, Mi G H, Zhang F S, et al. Estimation on nitrogen fertilizer saving potential of some summer maize hybrids in Beijing-TianjinTangshan area of China (in Chinese). J Maize Sci, 2009, 17: 115-117

23 Hallauer A R, Miranda J B. Quantitative Genetics in Maize Breeding. Ames: Iowa State University Press, 1981

24 Zhai W X, Tong L W. Agricultural Experimental Statistics Program BASIC. Shenyang: Liaoning Science and Technology Press, 1987. 
221-228

25 Gallais A, Coque M. Genetic variation and selection for nitrogen use efficiency in maize: a synthesis. Maydica, 2005, 50: 531-537

26 Anbessa Y, Juskiw P, Good A, et al. Selection efficiency across environments in improvement of barley yield for moderately low nitrogen environments. Crop Sci, 2010, 50: 451-457

27 Bänzinger M, Betran F J, Lafitte H R. Efficiency of high-nitrogen selection environments for improving maize for low-nitrogen target environments. Crop Sci, 1997, 37: 1103-1109

28 Presterl T, Seitz G, Landbeck M, et al. Improving nitrogen use efficiency in European maize: estimation of quantitative parameters. Crop Sci, 2003, 43: 1259-1265

29 Mi G H, Chen F J, Chun L, et al. Biological characteristics of nitrogen efficient maize genotypes (in Chinese). Plant Nutr Fert Sci, 2007, 13: $155-159$

30 Worku M, Bänzinger M, Erley G S, et al. Nitrogen uptake and utilization in contrasting nitrogen efficient tropical maize hybrids. Crop Sci, 2007, 47: 519-527

31 Coque M, Gallais A. genetic variation among European maize varieties for nitrogen use efficiency under low and high nitrogen fertilization. Maydica, 2007, 52: 383-397
32 Logroño M L, Lothrop J E. Impact of drought and low nitrogen on maize production in Asia. In: Edmeades, G O, Bänzinger M, Mickelson H R, et al., eds. Developing drought- and low N-tolerant maize. Proceedings of a Symposium, International Maize and Wheat Improvement Center, El Batán, Mexico. Mexico: International Maize and Wheat Improvement Center, 1996. 39-43

33 Machado A T, Fernandes M S. Participatory maize breeding for low nitrogen tolerance. Euphytica, 2001, 122: 567-573

34 Bänzinger M, Setimela P S, Hodson D, et al. Breeding for improved drought tolerance in maize adapted to southern Africa. In: Proceedings of the 4th International Crop Science Congress, Brisbane, Australia, 2004

35 Chen F J, Mi G H, Zhang F S, et al. Nitrogen use efficiency in some of main maize hybrids grown in North China (in Chinese). J Maize Sci, 2003, 11: 78-82

36 Chun L, Chen F J, Zhang F S, et al. Root growth, nitrogen uptake and yield formation of hybrid maize with different $\mathrm{N}$ efficiency (in Chinese). Plant Nutr Fert Sci, 2005, 11: 615-619

37 Presterl T, Groh S, Landbeck M, et al. Nitrogen uptake and utilization efficiency of European maize hybrids developed under conditions of low and high nitrogen input. Plant Breeding, 2002, 121: 480-486

Open Access This article is distributed under the terms of the Creative Commons Attribution License which permits any use, distribution, and reproduction in any medium, provided the original author(s) and source are credited. 